University of Wollongong

Research Online

Australian Institute for Innovative Materials -

Papers

Australian Institute for Innovative Materials

$1-1-2013$

Direct observation of domain wall motion and novel dielectric loss in 0.23Pb(In1/2Nb1/2)03-0.42Pb(Mg1/3 Nb2/3)03-0.35PbTiO3 crystals

Dabin Lin

Xi'an University of Technology

Zhenrong Li

Xi'an Jiaotong University

Fei Li

Xi'an Jiaotong University, lifei1216@gmail.com

Shujun Zhang

Xi'an Jiaotong University, shujun@uow.edu.au

Follow this and additional works at: https://ro.uow.edu.au/aiimpapers

Part of the Engineering Commons, and the Physical Sciences and Mathematics Commons

Research Online is the open access institutional repository for the University of Wollongong. For further information contact the UOW Library: research-pubs@uow.edu.au 


\title{
Direct observation of domain wall motion and novel dielectric loss in $0.23 \mathrm{~Pb}(\ln 1 /$ 2Nb1/2)03-0.42Pb(Mg1/3 Nb2/3)03-0.35PbTiO3 crystals
}

\author{
Abstract \\ Domain wall motion was directly observed at temperatures near to the monoclinic-to-tetragonal phase \\ transition temperature (TM-T) in [001]-oriented 0.23Pb(In1/2 Nb1/2)03-0. 42Pb(Mg1/3Nb2/ \\ 3)03-0.35PbTiO3 (0.23PIN-0.42PMN-0.35PT) single crystals using a polarizing light microscope, and \\ evident $90^{\circ}$ and $180^{\circ}$ domain switches were observed near the Curie temperature (TC). Two dielectric loss \\ anomalies were observed at temperatures near TM-T in the [001]-oriented PIN-PMN-PT single crystals, \\ while an additional dielectric loss peak was found at temperatures a few degrees below TC, which was \\ associated with domain wall motion. Based on the domain structure observations, a domain switching \\ mechanism was proposed to explain the novel dielectric loss peak at several degrees below TC. 2013 The \\ Royal Society of Chemistry.

\section{Keywords} \\ novel, dielectric, 3, 35pbtio3, crystals, motion, loss, wall, domain, observation, direct, 23pb, in1, 2nb1, 2, o3, \\ 42pb, mg1, nb2

\section{Disciplines} \\ Engineering | Physical Sciences and Mathematics

\section{Publication Details} \\ Lin, D., Li, Z., Li, F. \& Zhang, S. (2013). Direct observation of domain wall motion and novel dielectric loss in \\ $0.23 \mathrm{~Pb}(\operatorname{In} 1 / 2 \mathrm{Nb} 1 / 2) 03-0.42 \mathrm{~Pb}(\mathrm{Mg} 1 / 3 \mathrm{Nb} 2 / 3) 03-0.35 \mathrm{PbTiO3}$ crystals. CrystEngComm, 15 (32), \\ 6292-6296.
}


Cite this: CrystEngComm, 2013, 15, 6292

Received 3rd May 2013,

Accepted 5th June 2013

DOI: $10.1039 /$ c3ce40770e

www.rsc.org/crystengcomm

Domain wall motion was directly observed at temperatures near to the monoclinic-to-tetragonal phase transition temperature $\left(T_{\mathrm{M}-\mathrm{T}}\right)$ in [001]-oriented $0.23 \mathrm{~Pb}\left(\mathrm{In}_{1 / 2} \mathrm{Nb}_{1 / 2}\right) \mathrm{O}_{3}-0.42 \mathrm{~Pb}\left(\mathrm{Mg}_{1 / 3} \mathrm{Nb}_{2 / 3}\right) \mathrm{O}_{3}-$ $0.35 \mathrm{PbTiO}_{3}(0.23 \mathrm{PIN}-0.42 \mathrm{PMN}-0.35 \mathrm{PT})$ single crystals using a polarizing light microscope, and evident $90^{\circ}$ and $180^{\circ}$ domain switches were observed near the Curie temperature $\left(T_{\mathrm{C}}\right)$. Two dielectric loss anomalies were observed at temperatures near $T_{\mathrm{M}-\mathrm{T}}$ in the [001]-oriented PIN-PMN-PT single crystals, while an additional dielectric loss peak was found at temperatures a few degrees below $T_{\mathrm{C}}$, which was associated with domain wall motion. Based on the domain structure observations, a domain switching mechanism was proposed to explain the novel dielectric loss peak at several degrees below $T_{\mathrm{C}}$.

\section{Introduction}

Domain wall motion has a dominant position in the macroscopic response of ferroelectric and ferroelastic materials, for example, in the complex nonlinear response and significant hysteresis. In particular, it has been reported that the dielectric properties are closely related to domain wall motion in ferroelectric materials, ${ }^{1-3}$ where a dielectric loss peak has been observed at temperatures a few degrees below the Curie temperature $\left(T_{\mathrm{C}}\right)$, which is thought to be related to the movement of the domain walls. However, there is no direct observation of domain wall motion associated with the dielectric loss peak. It is one purpose of this research to study the domain wall evolution at various temperatures in order to understand the contribution of domain wall motion to the macroscopic dielectric properties.

Recently, $\mathrm{Pb}\left(\mathrm{In}_{1 / 2} \mathrm{Nb}_{1 / 2}\right) \mathrm{O}_{3}-\mathrm{Pb}\left(\mathrm{Mg}_{1 / 3} \mathrm{Nb}_{2 / 3}\right) \mathrm{O}_{3}-\mathrm{PbTiO}_{3} \quad$ (PINPMN-PT) ternary crystals with a higher $T_{\mathrm{C}}$ than that of $\mathrm{Pb}\left(\mathrm{Mg}_{1 / 3} \mathrm{Nb}_{2 / 3}\right) \mathrm{O}_{3}-\mathrm{PbTiO}_{3}$ binary crystals, and comparable high piezoelectric and dielectric properties near the morphotropic

${ }^{a}$ Laboratory of Thin Film Techniques and Optical Test, Xi'An Technological University, Xi'an 710032, China

${ }^{b}$ Electronic Materials Research Laboratory, Key Laboratory of Education Ministry, Xi'an Jiaotong University, Xi'an 710049, China.E-mail: zhrli@mail.xjtu.edu.cn; shujunzhang@gmail.com phase boundary (MPB) compositions, have attracted extensive investigations. ${ }^{4-6}$ The high piezoelectric coefficient $d_{15}$ in the single domain tetragonal PIN-PMN-PT crystals and the high extensional piezoelectric coefficients $d_{33} / d_{32}$ in the multidomain PIN-PMN-PT crystals were thought to be related to the domain state and polarization rotation. From the viewpoint of application, it is desirable to investigate the evolution of the domain structure at various temperatures in the PIN-PMN-PT crystals. In this work, the temperature induced phase transitions were investigated in the [001]-oriented $0.23 \mathrm{~Pb}\left(\mathrm{In}_{1 / 2} \mathrm{Nb}_{1 / 2}\right) \mathrm{O}_{3}-$ $0.42 \mathrm{~Pb}\left(\mathrm{Mg}_{1 / 3} \mathrm{Nb}_{2 / 3}\right) \mathrm{O}_{3}-0.35 \mathrm{PbTiO}_{3} \quad$ (0.23PIN-0.42PMN-0.35PT) crystals based on in-situ domain structure observations and dielectric/ferroelectric property measurements. A domain switching model was proposed to explain the electrical properties.

\section{Experimental}

PIN-PMN-PT crystals were grown using a modified Bridgman method. The studied composition was $0.23 \mathrm{PIN}-0.42 \mathrm{PMN}-0.35 \mathrm{PT}$, which is close to the monoclinic to tetragonal MPB region. ${ }^{7}$ The samples with sizes of $5 \times 5 \mathrm{~mm}^{2}$ were polished to $70 \mu \mathrm{m}$. The domain structure was observed using a polarizing light microscope (PLM) (Olympus BX51) with a LINKAM heating-cooling stage. Prior to the dielectric measurements, the samples were sputtered with transparent electrodes and poled at an electric field of $8 \mathrm{kV} \mathrm{cm}^{-1}$ at room temperature. The dielectric permittivity and dielectric loss were measured under a zero bias field as a function of temperature in the range of $20{ }^{\circ} \mathrm{C}$ to $250{ }^{\circ} \mathrm{C}$ at a heating rate of 3 ${ }^{\circ} \mathrm{C} \min ^{-1}$, by a multi-frequency LCR meter (HP4284A), which was connected to a computer controlled furnace. The polarization-field hysteresis loops were measured using a TF analyzer 2000 (aixACCT).

\section{Results and discussion}

The domain structure vs. temperature was investigated in [001]poled 0.23PIN-0.42PMN-0.35PT crystals by in-situ observations. The crystals presented two types of domains at $30{ }^{\circ} \mathrm{C}$, as shown in Fig. 1(a). One domain had domain walls parallel to the [010] 

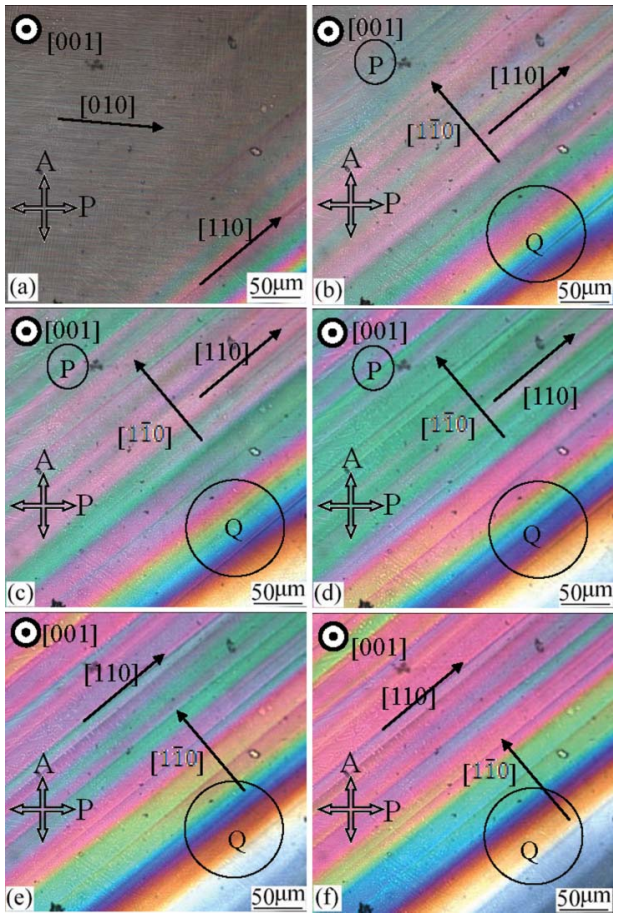

Fig. 1 The domain structures observed in the [001]-oriented 0.23PIN-0.42PMN$0.35 \mathrm{PT}$ crystals at temperatures of (a) $30{ }^{\circ} \mathrm{C}_{1}$ (b) $40{ }^{\circ} \mathrm{C}_{1}$ (c) $50{ }^{\circ} \mathrm{C}_{1}$ (d) $60{ }^{\circ} \mathrm{C}_{1}$ (e) 70 ${ }^{\circ} \mathrm{C}$ and (f) $80^{\circ} \mathrm{C}$

direction with a small domain size $(2 \mu \mathrm{m}-5 \mu \mathrm{m})$, and the other domain had domain walls parallel to the [110] direction with a large domain size $(20 \mu \mathrm{m}-50 \mu \mathrm{m})$. With a temperature increase (to $40{ }^{\circ} \mathrm{C}$ ), the small domain walls disappeared and new large domain walls parallel to the [110] direction were observed. Comparing the $\mathrm{P}$ and Q regions, as shown in Fig. 1(b)-(d), the non- $180^{\circ}$ domain walls changed with a temperature increase. Meanwhile, the interference colors also changed, as shown in Fig. 1(b)-(f), which may be associated with polarization rotation when the temperature increases from $40{ }^{\circ} \mathrm{C}$ to $80{ }^{\circ} \mathrm{C}$. As shown in Fig. $1(\mathrm{e})$ and (f), the large domain walls were slightly deviated from the [110] direction, and are related to the unstable polarization state at the temperature near the ferroelectric-to-ferroelectric phase transition.

The domain structure for the [001]-oriented 0.23PIN-0.42PMN$0.35 \mathrm{PT}$ crystals was investigated in the temperature range of 200 ${ }^{\circ} \mathrm{C}$ to $205{ }^{\circ} \mathrm{C}$, as shown in Fig. 2. Generally, the $180^{\circ}$ domain walls are not detected by the PLM because the polarization vectors are along the same direction in the poled samples. However, the $180^{\circ}$ polarization vectors are anti-parallel in the unpoled samples and the $180^{\circ}$ domain wall, which shows a black-white pattern, can be detected by the PLM. Comparing Fig. 2(a) and (b), the $180^{\circ}$ domain walls were detected at $202{ }^{\circ} \mathrm{C}$, which is indicative of the $180^{\circ}$ domain switch at this temperature. When the temperature was increased, the $90^{\circ}$ and $180^{\circ}$ domain coexisting region, marked as the $\mathrm{S}$ region, was broadened, as shown in Fig. 2(b)-(d). The colorful $90^{\circ}$ domain region changed to a black-white color, as shown in Fig. 2(e) and (f); meanwhile, new $180^{\circ}$ domain walls were found in Fig. 2(f). At $203.8{ }^{\circ} \mathrm{C}$, part of the domain structure disappeared, which is related to the ferroelectric-to-paraelectric

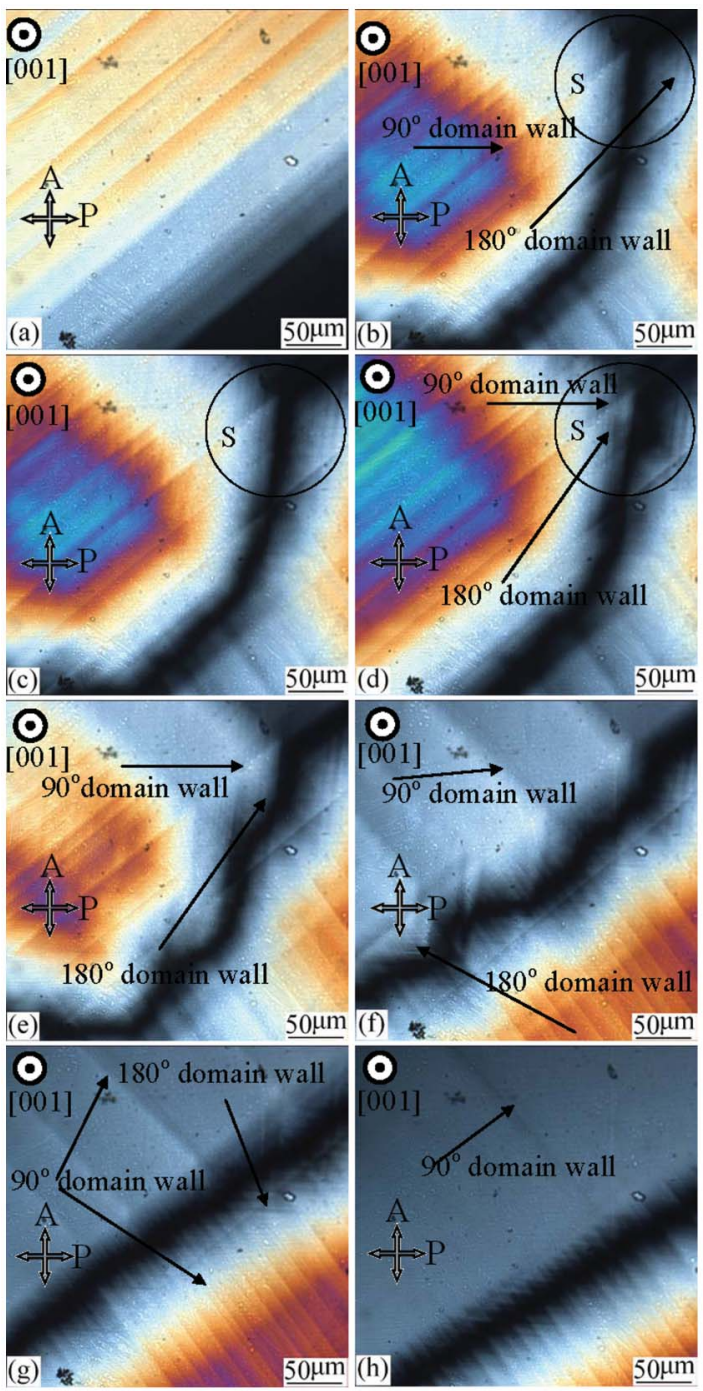

Fig. 2 The domain structures observed in the [001]-oriented PIN-PMN-PT crystals at temperatures of (a) $200{ }^{\circ} \mathrm{C}$, (b) $202{ }^{\circ} \mathrm{C}$, (c) $202.3{ }^{\circ} \mathrm{C}$, (d) $202.4{ }^{\circ} \mathrm{C}$, (e) $202.5^{\circ} \mathrm{C}$, (f) $202.6{ }^{\circ} \mathrm{C}$, (g) $203.8^{\circ} \mathrm{C}$ and (h) $204.1^{\circ} \mathrm{C}$.

phase transition, as shown in Fig. $2(\mathrm{~g})$. Comparing Fig. $2(\mathrm{~g})$ and (h), the number of $90^{\circ}$ and $180^{\circ}$ domain walls decreased when the temperature increased, and they totally disappeared at $205{ }^{\circ} \mathrm{C}$.

Fig. 3 shows the temperature dependence of the dielectric permittivity $\left(\varepsilon_{\mathrm{r}}\right)$ and dielectric loss $(\tan \delta)$ for the [001]-poled 0.23PIN-0.42PMN-0.35PT crystals under a zero bias field. Two dielectric permittivity peaks, peak I $\left(\sim 50{ }^{\circ} \mathrm{C}\right)$ and peak II $(\sim 207$ ${ }^{\circ} \mathrm{C}$ ), were observed in the poled samples. Peak I is the $\mathrm{M}_{\mathrm{C}}$-to-T phase transition, while peak II reflects the T-to-C phase transition. Two distinct dielectric loss peaks, peak I-I $\left(\sim 60^{\circ} \mathrm{C}\right)$ and peak I-II $\left(\sim 80{ }^{\circ} \mathrm{C}\right)$, were observed at temperatures higher than $T_{\mathrm{M}-\mathrm{T}}$. Correspondingly, the dielectric permittivity curve shows a step-like decrease when the temperature increases from $60{ }^{\circ} \mathrm{C}$ to $80^{\circ} \mathrm{C}$. It is of particular interest that some other step-like dielectric loss anomalies were found before peak I-I, which correspond to the $\mathrm{M}_{\mathrm{C}}$-to-T phase transition. Furthermore, two novel dielectric loss peaks were detected at $201{ }^{\circ} \mathrm{C}$ and $207{ }^{\circ} \mathrm{C}$, respectively. Moreover, 


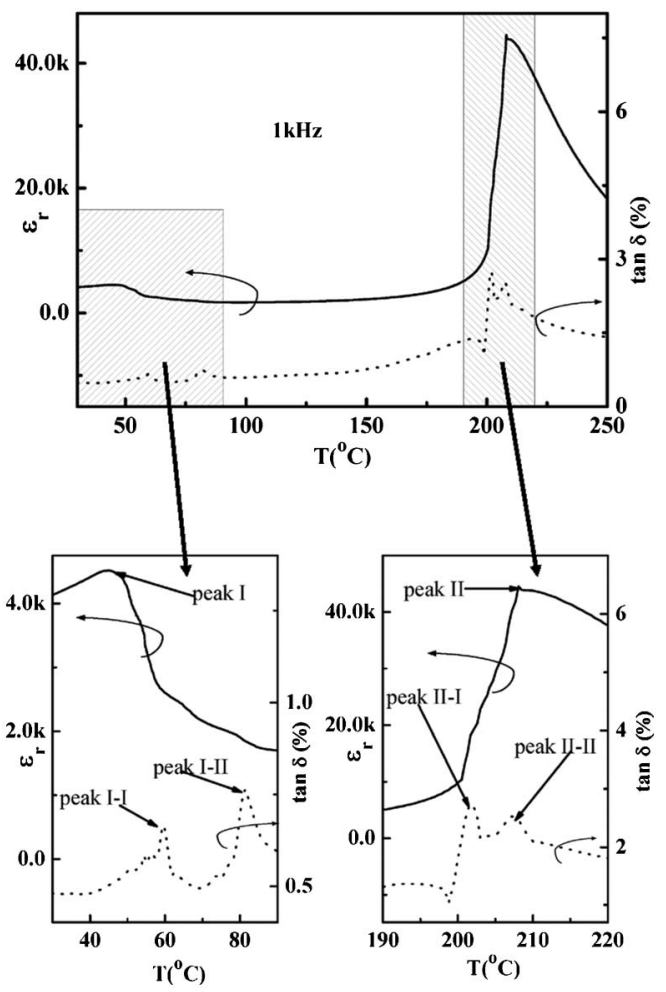

Fig. 3 The dielectric permittivity and dielectric loss measured as functions of temperature in the [001]-oriented 0.23PIN-0.42PMN-0.35PT crystals.

the dielectric permittivity curve showed a step-like increase from $201{ }^{\circ} \mathrm{C}$ to $207{ }^{\circ} \mathrm{C}$.

Two dielectric permittivity peaks corresponding to the $\mathrm{R}-\mathrm{M}$ and $\mathrm{M}-\mathrm{T}$ phase transitions, were detected in the [001]-poled rhombohedral PMN-0.30PT crystals. ${ }^{8,9}$ Meanwhile, two dielectric loss peaks, one associated with the R-to- $\mathrm{M}_{\mathrm{A}}$ phase transition and one associated with the $\mathrm{M}_{\mathrm{A}}$-to- $\mathrm{M}_{\mathrm{C}}$ phase transition, indicating a two-step change were found near $T_{\mathrm{R}-\mathrm{M}} \cdot{ }^{8,9}$ However, only one permittivity peak $\left(\sim 50{ }^{\circ} \mathrm{C}\right)$ was observed near $T_{\mathrm{M}-\mathrm{T}}$ for the [001]poled 0.23PIN-0.42PMN-0.35PT crystals, as shown in Fig. 3. Furthermore, no dielectric loss peak was observed below $T_{\mathrm{M}-\mathrm{T}}$. Based on the dielectric property analysis, the $\mathbf{M}_{\mathrm{C}}$ phase was thought to dominate the [001]-poled 0.23PIN-0.42PMN-0.35PT crystals at room temperature. The phase transition path of $\mathrm{R} \rightarrow$ $\mathrm{M}_{\mathrm{A}} \rightarrow \mathrm{M}_{\mathrm{C}} \rightarrow \mathrm{T}$ has been confirmed in the [001]-oriented PMN-PT crystals in an electric field, based on XRD and neutron diffraction scattering research. ${ }^{10}$ In addition, the stable monoclinic phase has been reported in the [001]-poled PMN-0.30PT crystals, based on the dielectric measurements. ${ }^{8}$ That is to say, the polarization rotated to the [001] direction. Based on the domain observations and the dielectric behavior, the polarization rotation path was $\mathrm{M}_{\mathrm{C}^{-}}$ $\mathrm{T}$ in the [001]-oriented 0.23PIN-0.42PMN-0.35PT crystals with an electric field applied along the [001] direction.

Direct domain wall motion was observed in the [001]-poled 0.23PIN-0.42PMN-0.35PT crystals, as shown in Fig. 1 and 2. In order to confirm the factor contributing to domain wall motion, the polarization hysteresis loops were measured at increasing temperatures for the $0.23 \mathrm{PIN}-0.42 \mathrm{PMN}-0.35 \mathrm{PT}$ crystals. Fig. 4 shows the temperature dependent (a) remnant polarization $\left(P_{\mathrm{r}}\right)$ and (b) coercive field $\left(E_{\mathrm{c}}\right)$. The $P_{\mathrm{r}}$ and $E_{\mathrm{c}}$ were found to be $28 \mu \mathrm{C}$ $\mathrm{cm}^{-2}$ and $6.3 \mathrm{kV} \mathrm{cm}^{-1}$ in the [001]-oriented 0.23PIN-0.42PMN$0.35 \mathrm{PT}$ crystals at room temperature, respectively. $P_{\mathrm{r}}$ exhibited a clear drop near $40{ }^{\circ} \mathrm{C}$, implying a significant polarization reorientation of the local domains. This is consistent with the $\mathrm{M}_{\mathrm{C}}-\mathrm{T}$ phase transition temperature, as shown in Fig. 3 , and the evident domain wall motion in Fig. 1(a) and (b). With an increase in temperature, the polarization vector of $\mathrm{M}_{\mathrm{C}}$ rotated to $\mathrm{T}$ below $T_{\mathrm{M}-\mathrm{T}}$, while some other polarization vector turned back to $\mathrm{M}_{\mathrm{C}}$ because of the unstable polarization state. Therefore, non- $180^{\circ}$ domain wall motion was observed when the temperature increased from $40{ }^{\circ} \mathrm{C}$ to $50{ }^{\circ} \mathrm{C}$, as shown in Fig. 1(a)-(c), which was induced by the polarization rotation. When the temperature was further increased, the polarization vector totally turned to the [001] direction due to the $\mathrm{M}_{\mathrm{C}}$-to-T phase transition, and clear non$180^{\circ}$ domain wall motion could be observed at temperatures from $50{ }^{\circ} \mathrm{C}$ to $80{ }^{\circ} \mathrm{C}$, as shown in Fig. 1(c)-(f). Based on the above analysis, it can be concluded that the domain wall motion observed in Fig. 1 was induced by polarization rotation.

In the in-situ domain observations as a function of temperature for the [001]-oriented 0.23PIN-0.42PMN-0.35PT crystals, the small domain structures were found to disappear at $40{ }^{\circ} \mathrm{C}$, while domain wall motion was observed from $40{ }^{\circ} \mathrm{C}$ to $80{ }^{\circ} \mathrm{C}$. In the dielectric measurements, a dielectric permittivity peak was detected at $50{ }^{\circ} \mathrm{C}$, which showed a step-like decrease when the temperature increased from $60{ }^{\circ} \mathrm{C}$ to $80{ }^{\circ} \mathrm{C}$. Furthermore, step-like dielectric loss anomalies were found from $30{ }^{\circ} \mathrm{C}$ to $50{ }^{\circ} \mathrm{C}$, and two distinct dielectric loss peaks were observed from $60{ }^{\circ} \mathrm{C}$ to $80{ }^{\circ} \mathrm{C}$. Comparing the results from the domain observations and dielectric properties, the change in the small domain structure was thought to be associated with the $\mathrm{M}_{\mathrm{C}}-\mathrm{T}$ phase transition; moreover, the domain wall motion contributed to the dielectric loss and dielectric permittivity. The non- $180^{\circ}$ domain walls contributed to the dielectric properties, which is similar to previous research. ${ }^{11,12}$ In the PMN-PT crystals, no additional dielectric loss peak was observed at temperatures higher than $T_{\mathrm{M}-\mathrm{T}}$, which is due to the restriction of the domain wall motion by the external dc field. In the present work, there was no more phase transition between the $\mathrm{M}_{\mathrm{C}}$ and $\mathrm{T}$ phases in the PINPMN-PT crystals. Therefore, it is reasonable to conclude that the additional dielectric loss peaks and step-like dielectric permittivity behavior in the [001]-oriented 0.23PIN-0.42PMN$0.35 \mathrm{PT}$ crystals were induced by the domain wall motion, and the domain wall motion was related to the unstable polarization state through the polarization rotation.

On the other hand, it has been reported that a dielectric loss peak exists at temperatures a few degrees below $T_{\mathrm{C}}$ in ferroelectric materials such as KDP, PMN-PT and PIN, ${ }^{1-3}$ which was thought to be related to domain wall motion. In this work, direct $90^{\circ}$ domain wall motion was observed from $200{ }^{\circ} \mathrm{C}$ to $205{ }^{\circ} \mathrm{C}$; meanwhile, an additional dielectric loss peak was found at $201{ }^{\circ} \mathrm{C}$. It is of particular importance that the $180^{\circ}$ domain switch was detected at $202^{\circ}$, as shown in Fig. 2, and the $90^{\circ}$ domain wall motion was found to be driven by the $180^{\circ}$ domain switching. Based on the results of the domain observations and dielectric properties, it can 

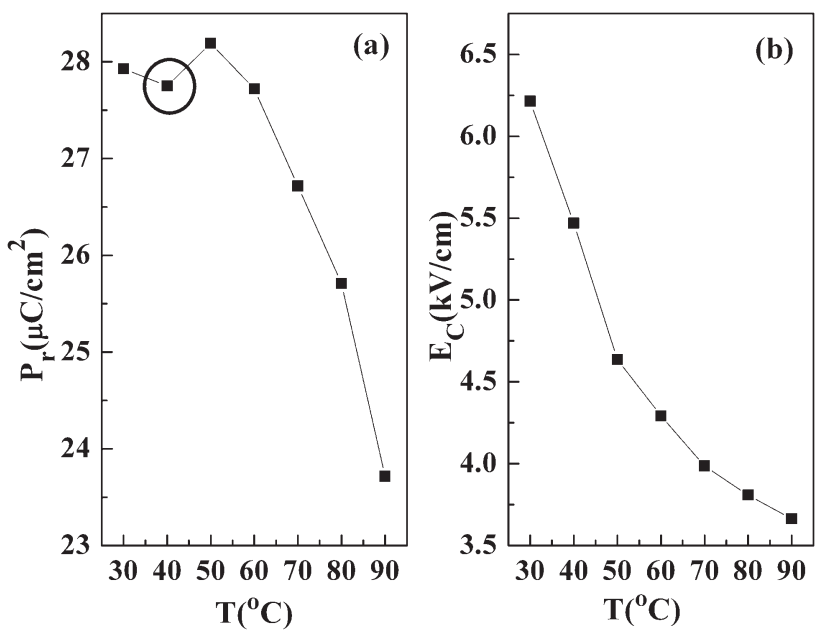

Fig. 4 The temperature-dependent (a) remnant polarization $\left(P_{\mathrm{r}}\right)$ and $(\mathrm{b})$ coercive field $\left(E_{c}\right)$ for the [001]-oriented 0.23PIN-0.42PMN-0.35PT crystals.

be concluded that the abnormal dielectric loss near the ferroelectric-to-paraelectric phase transition was related to the $180^{\circ}$ domain switching and $90^{\circ}$ domain wall motion.

Based on the domain structure observations, a $90^{\circ}$ and $180^{\circ}$ domain co-switching mechanism was proposed to explain the new domain observation near the ferroelectric-to-paraelectric phase transition, as given in Fig. 5. A description of the $90^{\circ}$ and $180^{\circ}$ domain walls in the tetragonal PIN-PMN-PT crystal is given in

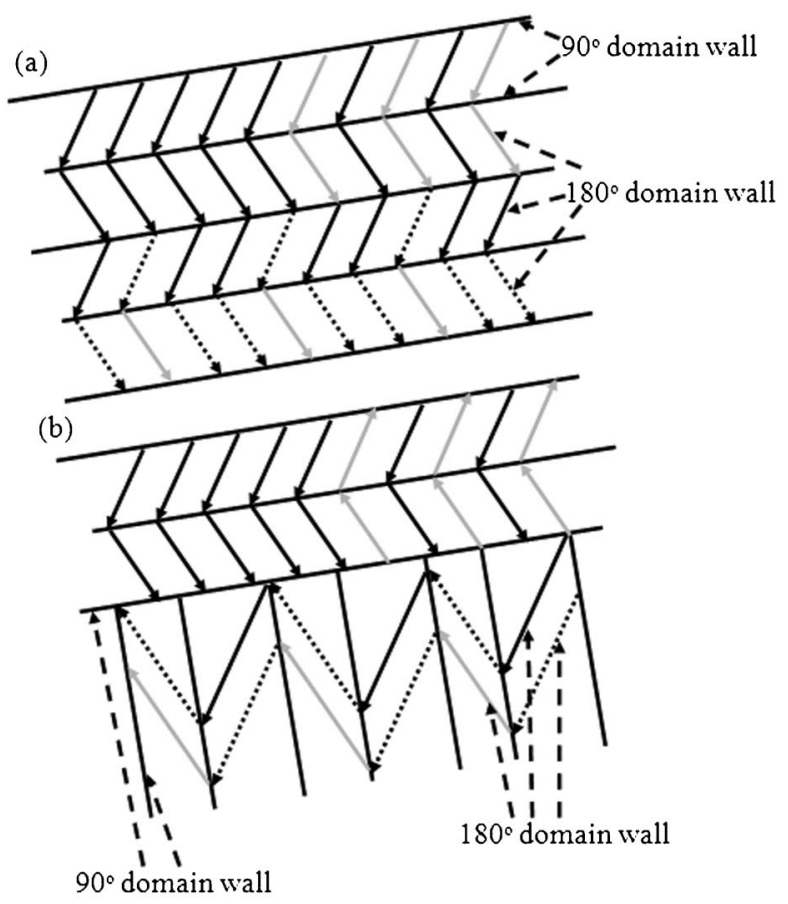

Fig. 5 Schematics of the $90^{\circ}$ and $180^{\circ}$ domain co-switching mechanism near the ferroelectric-to-paraelectric phase transformation. The paralleled lines represent the $90^{\circ}$ domain walls; the solid and dotted arrows represent the 180 domain walls.
Fig. 5(a), while the change of the domain walls at temperatures below the Curie temperature is shown in Fig. 5(b). In accordance with the domain observations, the $180^{\circ}$ domain walls were not detected by the PLM because of the parallel polarization vector. The $180^{\circ}$ domain walls were detected by the PLM at $202{ }^{\circ} \mathrm{C}$, which indicated that part of the $180^{\circ}$ domains reversed and became antiparallel to the other $180^{\circ}$ domains. Moreover, the parallel $90^{\circ}$ domain walls shown in Fig. 2(a) were separated by $180^{\circ}$ domain walls, and presented a vertical shape as shown in Fig. 2(b). On further increasing the temperature, the $90^{\circ}$ domain walls rotated $90^{\circ}$ and started to disappear. In the proposed model, partial $180^{\circ}$ domains, marked with gray arrows, are reversed and become antiparallel to their original direction, while other $180^{\circ}$ domains, marked with dotted arrows, make a $90^{\circ}$ rotation. As a result, the $90^{\circ}$ domain walls are switched, which is due to the $180^{\circ}$ domain wall motion. This model is consistent with the domain observations shown in Fig. 2, and can explain the new dielectric loss peak shown in Fig. 3.

\section{Conclusions}

Non- $180^{\circ}$ domain wall motion was observed at temperatures near $T_{\mathrm{M} \text {-T }}$, while $180^{\circ}$ domain switching near $202^{\circ} \mathrm{C}$ (slightly lower than $T_{\mathrm{C}}$ ) was observed using a PLM. The domain wall motion induced by polarization rotation was confirmed by in-situ domain observations and dielectric measurements, and gave rise to the two new dielectric loss peaks at temperatures a few degrees above the $T_{\mathrm{M}-\mathrm{T}}$. In addition, a $90^{\circ}$ and $180^{\circ}$ domain co-switching mechanism was employed to explain the domain wall motion at temperatures a few degrees below $T_{\mathrm{C}}$.

\section{Acknowledgements}

This work was supported by the National Natural Science Foundation of China No. 50872106 and No. 50632030, the National Basic Research Program of China (973 Program) No. 2009CB623306, the International Science \& Technology Cooperation Program of China No. 2010DFR50480 and the National Nature Science Foundation of China-NSAF No. 10976022.

\section{References}

1 Y. N. Huang, Y. N. Wang and H. M. Shen, Phys. Rev. B: Condens. Matter, 1992, 46, 3290.

2 S. W. Choi, T. R. Shrout, S. J. Jing and A. S. Bhalla, Ferroelectrics, 1989, 100, 29.

3 A. A. Bokov and V. Y. Shonov, Ferroelectrics, 1990, 108, 237.

4 F. Li, S. Zhang, Z. Xu, X. Wei, J. Luo and T. R. Shrout, J. Appl. Phys., 2010, 107, 054107.

5 S. Zhang and F. Li, J. Appl. Phys., 2012, 111, 031301.

6 F. Li, S. Zhang, D. Lin, J. Luo, Z. Xu, X. Wei and T. R. Shrout, J. Appl. Phys., 2011, 109, 014108.

7 D. Lin, Z. Li, F. Li, Z. Xu and X. Yao, J. Alloys Compd., 2010, 489, 115.

8 D. Lin, Z. Li, S. Zhang, Z. Xu and X. Yao, J. Appl. Phys., 2010, 108, 034112. 
9 Z. Li, Z. Xu, X. Yao and Z.-Y. Cheng, J. Appl. Phys., 2008, 104, 024112.

10 F. M. Bai, N. G. Wang, J. F. Li, D. Viehland, G. Xu and G. Shirane, J. Appl. Phys., 2004, 96, 1620.
11 G. Arlt, H. Dederichs and R. Herbiet, Ferroelectrics, 1987, 74, 37. 12 R. Herbiet, U. Robels, H. Dederichs and G. Arlt, Ferroelectrics, 1989, 98, 107. 